Revista Digital Año 8. No 10 - Año 2017. -pág. 1-151

ISSN 1853-1393

Resistencia. Chaco. Argentina - 2017

\title{
LA FORMACIÓN DOCENTE PARA LA CIUDADANÍA EN CONTEXTOS DE NEOLIBERALISMO: PROPUESTAS DESDE LAS NARRATIVAS
}

\section{TEACHER TRAINING FOR CITIZENSHIP IN CONTEXTS OF NEOLIBERALISM: PROPOSALS FROM THE NARRATIVES}

\author{
Marta Susana Bertolini ${ }^{1}$
}

Fecha de recepción: 24-11-2016

Fecha de aceptación y versión final: 28-04-2017

\begin{abstract}
Resumen
Las políticas neoliberales expandidas a nivel global afectan el ejercicio de los derechos y de una ciudadanía plena. El Proyecto de Investigación del que forma parte este trabajo, busca comprender los modos en que las estudiantes del profesorado de educación inicial viven lo político, situaciones que recuperan desde su biografía escolar y sus historias de vida, a fin de visibilizarlas y analizar los contextos de producción, para desde la concienciación y reflexividad, reconocerse como sujetos activos en la construcción de su propia subjetividad y en la construcción del mundo que habitan, en sus dimensiones sociales, económicas, políticas y culturales, es decir posicionarse como sujetos activos de la política. Al relatar la propia experiencia, a través de la narrativa, es posible visibilizar la constitución de la propia subjetividad y tomar consciencia de los mundos que nos habitan, distinguiendo diferentes técnicas de dominación. Experiencias, éstas, indispensables para realizar propuestas pedagógicas en las que desde los primeros meses de vida los niños y las niñas sean partícipes reales de procesos de socialización identificados como emancipatorios.
\end{abstract}

Palabras clave: Formación docente - neoliberalismo — narrativa - educación infantil

\begin{abstract}
Neoliberal policies expanded globally affect the exercise of rights and full citizenship. The Research Project of which this work is part, seeks to understand the ways in which the students of the initial education teachers live the political, situations that they recover from their school biography and their life stories, in order to make them visible and analyze the contexts of production, from the awareness and reflexivity, to recognize themselves as active subjects in the construction of their own subjectivity and in the construction of the world they inhabit, in their social, economic, political and cultural dimensions, that is, to position themselves as active subjects of politics. By relating our own experience, through the narrative, it is possible to visualize the constitution of our own subjectivity and to become aware of the worlds that inhabit us, distinguishing different techniques of domination. Experiences, these, indispensable to make pedagogical proposals in which, from the first months of life, boys and girls are real participants in socialization processes that are identified as emancipatory.
\end{abstract}

Key words: Teacher training - neoliberalism - narrative - children's education

\footnotetext{
${ }^{1}$ Egresada de la Universidad Nacional del Nordeste con los títulos de Profesora en Educación Pre elemental, Profesora en Ciencias de la Educación y Licenciada en Ciencias de la Educación. Especialista en Curriculum y Prácticas Escolares, FLACSO -Actualmente cursando Doctorado en Filosofía. Profesora Adjunta a cargo por concurso en las cátedras Didáctica en la Educación Inicial y Taller de Integración, Investigación y Práctica III ambas de las carreras de Profesorado. Licenciatura en Educación Inicial de la Facultad de Humanidades - UNNE- Domicilio: Mendoza 728 - Resistencia - Chaco Celular: 3624155124. Correos: martasbertolini@hotmail.com; martasbertolini@gmail.com.
} 


\section{Introducción}

Este trabajo presenta algunos avances y reflexiones que venimos haciendo como integrantes del proyecto La formación de docentes en educación infantil entre el neoliberalismo y la construcción de ciudadanía, aprobado por Resolución 678/13 del Consejo Superior de la Universidad Nacional del Nordeste. Dentro del grupo de investigación -FODEI- abordamos la formación de docentes en la educación infantil, especialmente desde o en sus perspectivas ético políticas y pedagógicas. En mi caso en particular trabajando con las experiencias que las estudiantes del tercer nivel de las carreras de profesorado y licenciatura en educación inicial, han vivido respecto a la construcción de ciudadanía. El trabajo se inscribe en una metodología cualitativa y se sostiene ideológicamente en la convicción de que trabajar con y desde las identidades favorece la resistencia activa a los procesos de "des subjetivación" (Gerez Ambertin, 2009) que desde hace un tiempo las políticas del mercado con su lógica consumista y cosificadora promueven. Sin ninguna duda el re encuentro de cada sujeto con su propia historia, propicia un anclaje en sí mismo, desde donde se hace posible retomar los análisis de los diversos contextos, los discursos y prácticas hegemónicos, y develar desde esas mismas prácticas, las situaciones de disciplinamiento y control, que el patriarcado, el colonialismo y el capitalismo, entre otras prácticas fomentan (Sousa Santos, 2017) posibilitando la apertura de procesos de resistencia a las mismas. Cuando hablamos de perspectivas ético políticas, lo hacemos siguiendo a Carlos Cullen y entendiendo a la educación y a la formación docente como una acción, en las que, la libertad es parte constitutiva de ambas. Educación y formación docente que deben ser transformadoras y liberadoras, orientadas por el sentido de justicia involucrando necesariamente a los derechos humanos.

Algunas de las preguntas sobre las que hemos trabajado y que forman parte del Proyecto de Investigación son: ¿Cómo construyen los y las estudiantes del profesorado su subjetividad de ciudadanos? ¿Cuáles son las experiencias de la formación que les permiten pensarse y actuar como ciudadanos? ¿Qué características tienen las experiencias vinculadas a los procesos de construcción de ciudadanía que viven las y los estudiantes? ¿Cuáles son los rasgos de la historia personal de las y los estudiantes que se visibilizan en su proceso de formación? ¿Qué tipo de situaciones viven las y los estudiantes en su proceso de formación, que resultan significativas para la misma?. ¿Cuáles son las caracterísiticas de la cultura organizacional e institucional que contextualizan las experiencias de formación?.¿Cómo se relacionan en la propia construcción subjetiva las experiencias de formación con las condiciones organizacionales en que se realizan?

\section{Haciendo un poco de historia}

Presentar la historia de la cátedra Taller de Integración, Investigación y Práctica III, de dictado anual, y dentro de ella, nuestras propias preocupaciones, tiene que ver con decisiones metodológicas, ideológicas y epistemológicas. (Rivas Flores, 2012)

Nuestro interés se centra en comprender el modo en que los sujetos construyen sus identidades en relación con los contextos socio culturales (familiares, políticos, económicos, laborales, recreativos, etc.) en que viven, Cada biografía pone de manifiesto ésta complejidad, al tiempo en que presenta el modo en el que cada sujeto construye y elabora su propia vida. (Rivas Flores, 2012, p.115)

Cabe destacar que es en atención a esta perspectiva que se presenta una reseña que da cuenta del contexto en el cual se inicia el Proyecto y que sin duda 
marca su propio devenir. Al decir de Candau (2001), "el trabajo de la memoria, es entonces una mayéutica de la identidad, siempre renovada en cada narración".

La cátedra forma parte del Plan de Estudios 2000, así identificado porque es el año en que se aprueba el mismo, comenzando a regir desde el 2001. Es así que el 2003 es el primer año en que se dicta el Taller, acomodamientos personales, institucionales y profesionales van haciendo emerger hacia el año 2010, primero bastante tímidamente, pero luego con mayor fuerza, la definición de un eje transversal que más allá que fuimos cambiando su enunciado siempre giró en torno a la formación docente, el ejercicio de derechos y la ciudadanía enfatizando la propuesta de abordaje de la socialización política como eje de la educación de los niños y de las niñas.

En el año 2011 inicio una ponencia con una frase con la que termina su defensa de Tesis una alumna de la Licenciatura en Educación Inicial

Los docentes deberían preocuparse no sólo de enriquecer el currículum con contenidos científicos y tecnológicos, sino ampliarlo a la enseñanza de formas de actuar en lo humano y en los sistemas de relaciones que hacen referencia a las actitudes, normas y valores necesarios para una sociedad más justa y sólida." Terradillo (2010)

Esta afirmación se condice con la propuesta de Sara Alvarado (2009) que vincula la formación docente y la enseñanza en las instituciones educativas con las condiciones sociales, políticas, económicas en que se concretan estos procesos y también en la influencia que sobre la formulación del plan de estudios vigente tuvieron las políticas neoliberales de esos años y que se tradujo en escasos espacios curriculares destinados a abordar la problemática social y el abordaje desde la perspectiva de derechos humanos a lo que se suma que la mayoría de los estudiantes que en ese momento cursaban el Taller, (situación que no ha cambiado e incluso se ha profundizado) han sido ellos mismos blanco de esas políticas, en las que las relaciones sociales se entretejen y se resuelven a partir de la oferta y la demanda. Desde estas reflexiones presentamos en ese momento diferentes estrategias, incorporando el trabajo con las biografías escolares y con la posibilidad de desnaturalizar las relaciones sociales y los diversos aspectos que en ellas se juegan. Aquí surge la primera cuestión que nos moviliza cuando las estudiantes manifiestan su primera dificultad traducida en la imposibilidad de hacer preguntas dirigidas a la comprensión de la realidad social; de las entrevistas surge que con lo que se sienten cómodas y pueden hacer es "elaborar respuestas", respuestas que siempre estuvieron destinadas a satisfacer al maestro o al profesor, o al menos dicen que responden lo que así creían. Al retornar a este trabajo y a las vivencias de los estudiantes pienso en la situación de enajenamiento que la reproducción del sistema capitalista y sus modos de sometimiento produce, ya que salvo contadas excepciones, las estudiantes ponen en evidencia la lógica de lo escolar con sus grandes cuotas de homogeneización, disciplinamiento y silenciamiento de voces; el funcionamiento de las lógicas de "lo escolar" se ha planteado para el Jardín de infantes, la escuela primaria, la escuela secundaria y aún para algunos espacios dentro de la formación universitaria. La segunda de las cuestiones que señalamos en ese trabajo es el desconcierto de las estudiantes al proponerles algunos márgenes de libertad para seleccionar bibliografías para ir trabajando en los informes relacionados a la indagación sobre la realidad y los cuestionamientos que surgen de ellos mismos por considerar poco apropiado que los estudiantes accedan a diversos contenidos, es decir, parecería que surge cierta incomodidad cuando se le proponen situaciones que rompe la homogenización del grupo clase, que tendría que ver con la complejidad que impone pensar "lo escolar" (Baquero, Diker y Frigerio, 2007). Cerramos esa comunicación con una cita de Carlos Cullen 
que al plantear la posibilidad de "construir narrativamente la identidad profesional del maestro" continúa interpelándonos planteando tres escenarios educativos de la formación docente

Enseñar y aprender a tomar la palabra, en tiempos de retirada de la palabra, como modo de ingresar al espacio público.(...) aumentar la potencia de actuar, en tiempos de pensamiento único, como modo de hacer la "diferencia ética" (...) [y] resistir con inteligencia responsable, en tiempos de disciplinamiento y biopoder, como modo de cuidar la subjetividad, frente a todo intento de suprimirla, o borrarla o limitarla, pero siempre desde la interpelación del otro, que nos hace responsables y nos desilusiona de creernos "invulnerables.(Cullen, 2009,p. 15)

En el año 2012 presentamos un trabajo en las $\mathrm{V}$ Jornadas Nacionales de Prácticas y Residencias en la Formación Docente que se realizaron en la ciudad de Córdoba en el que recuperamos las voces de las estudiantes respecto a su propio proceso de formación. Algunas expresiones que recogimos en ese momento fueron:

Me resultó significativo el poder leer que la formación es un proceso personal, de comprensión y reflexión por cuenta propia, la cual resulta imprescindible para la realización de una buena práctica profesional. Pero, sin embargo, en un primer momento, no me daba cuenta de ello ya que pensaba que solo con contar con las teorías adecuadas podría enfrentar en cualquier situación; pero ahora sostengo que es necesaria la realización de un trabajo sobre mi misma contando con la ayuda de mediadores, que me puedan permitir elaborar mi propia forma para ejercer mi profesión. Estudiante A, 2012

Estas prácticas y planificarlas me ayudaron a darme cuenta de que en realidad la carrera que había elegido para mi futuro no es fácil, requiere de esfuerzos y además si no tenés la capacidad de reflexionar durante la situación áulica, luego puede ser tarde. Inclusive me di cuenta que me vivía quejando por todo lo que tenía que estudiar, por tener que ir a los jardines y me molestaba llegar ahí y encontrarme con docentes que me digan que no entendía o no sabía nada. Pero en realidad, pude entender que son este tipo de cosas, las que te dan más fuerzas para pelear por la educación y re significación de los niños. Estudiante R, 2012

\section{Finalizamos la comunicación expresando}

Nos damos cuenta de que la mayoría ve en sí lo que querría ver y no siempre lo que realmente está siendo, la formación requiere poner en cuestión además de las propias teorías implícitas los tipos de racionalidad con las que fueron elaboradas y son sostenidas para lo cual aún no tenemos nosotras mismas estrategias. Por eso deseamos participar de estas Jornadas, necesitamos la voz, las voces de otros que, con otras miradas, diversas perspectivas, nos ayuden a pensar... (Bertolini, Gonzalez, Alonso, 2012)

El año 2013 nos encuentra profundizando el concepto de experiencia, ya que es al que le brindamos atención muy especial a partir de la lectura de Ferry (1990) sobre los procesos de formación. En la ponencia La formación docente: entre el capitalismo y la ciudadanía plena, presentada en las $7^{\circ} \mathrm{Jornadas}$ Nacionales sobre la Formación del profesorado "Currículo, Investigación y Prácticas en contexto(s)" organizadas por la Facultad de Humanidades de la Universidad Nacional de Mar del Plata, recuperamos los aportes de Larrosa $(2003,2009)$ y en esta memoria, nos resultan particularmente significativas las palabras de "singularidad" "irrepetibilidad", "pluralidad", "pasión", "receptividad primera", "disponibilidad fundamental" y "apertura esencial" que el autor vincula a 
"experiencia". En el trabajo mencionado decíamos que los quiebres, los "dolores", las incertidumbres que la experiencia nos genera "tienen que ver con la resistencia, con la dignidad de hacerse cargo de uno mismo, de ampliar sus propios horizontes". En esta dirección continuamos trabajando hasta la fecha. Así como en el primer trabajo que aquí recuperamos detectamos las dificultades en problematizar la realidad y más concretamente en hacer preguntas que permitan procesos de problematización y des naturalización de la vida cotidiana y del ejercicio de la profesión docente, aquí recuperamos el "descubrimiento" que de alguna manera realizan las estudiantes sobre los procesos de formación que requiere de reflexión permanente sobre uno mismo. En esta misma comunicación, presentamos los resultados de nuestra indagación sobre los sentidos que las estudiantes le otorgan al término "prácticas pedagógicas" y advertimos que alrededor del $90 \%$ se circunscriben a los siguientes conceptos:

"a. $\quad$ poder llevar al campo porque es ahí que se podrán verificar todo lo que se aprendió desde el primer momento y poder volcarlo en las prácticas del jardín con los alumnos/as.

b. poder poner en práctica todos esos conocimientos e información adquirido a través de la integración, la investigación dentro del lugar de trabajo. Es allí donde uno se da cuenta si te sirvió todos los pasos hasta llegar hasta aquí.

c. entiendo que vamos al campo, es decir, vamos a la realidad a tratar de poder ver esa realidad y poder interpretarla y actuar con las herramientas que contamos.

d. momento en el cual se vuelca en la realidad lo aprendido por los diferentes autores de un modo crítico, responsable y adecuado al contexto real que nos toque presenciar, como también valorando lo dialogado en las diferentes clases, es decir el momento de práctica es una integración entre lo investigado desde los autores y el dialogo presente en el taller.

e. llevar a cabo todo lo aprendido teóricamente"

Hoy, año 2017, indagando sobre el mismo tema, las respuestas siguen siendo prácticamente las mismas, el $80 \%$ del grupo total sostiene que "es poner en juego los saberes obtenidos","es la acción que realizamos a partir de la teoría que estudiamos", "es poder llevar a la práctica todos los temas y contenidos que se dan en el aula (de la Facultad)", "la oportunidad de poner en práctica los temas abordados", "a través de las prácticas se ponen en juego los conocimientos adquiridos", "realizar acciones en base a los conocimientos adquiridos", "volcar nuestros conocimientos en la sala de Jardín junto con los niños", "es el lugar donde lo teórico es volcado a la práctica"; del $20 \%$ restante algunas estudiantes no contestan, otras sostienen que las prácticas "sirven para aprender, para equivocarse, para crecer", "es compartir experiencias", "para comprender la realidad", "es lo que nos va a permitir conocer nuestro campo". Al solicitarles la reflexión para de alguna manera identificar algunos aspectos del "universo simbólico" desde el cual hablan y la reflexión sobre la propia subjetividad surgen las vinculaciones con lo vivido como alumnas. Siguiendo a Rivas Flores (2012: 127) el propósito central de todas estas reflexiones es "sacar a la luz" estas "teorías" (identidad) que los estudiantes asumen y ponerlas en relación con los contextos en que fueron elaborándose. En este sentido surge claramente el fuerte protagonismo que el alumno practicante le da a su presencia en las aulas de las instituciones de educación infantil, la ausencia casi absoluta de visibilización de los niños y de las niñas, es decir de aquéllos a quienes está destinado el quehacer pedagógico, el acento fuertemente tecnocrático que se desprende de sus expresiones que 
considera la neutralidad del hecho educativo y por lo tanto no reconoce las vinculaciones que se dan con los aspectos políticos, sociales, económicos y culturales del contexto y que atraviesan cualquier práctica social y por supuesto la pedagógica. El "pensar epistémico" que nos propone Zemelman (2005) como esta posibilidad de "distanciarse de los constructos" para elaborar problemas que aporten miradas o perspectivas diferentes a las proposiciones teóricas a las que la realidad siempre desborda, ayuda a re construir los relatos desde posicionamientos que pretendemos sean críticos.

En un trabajo que elaboramos en el año 2014, La formación docente: análisis de los núcleos conceptuales a partir de la propias narrativas, y que fuera presentado en las VI Jornadas Nacionales de Prácticas y Residencias en la Formación Docente realizadas en la ciudad de Córdoba presentamos otra cuestión que surgió en el momento de realizar el período de observaciones y prácticas y es la disociación no percibida por ellas, entre las teoría consideradas pertinentes y su propio accionar, fragmentación que lleva a afirmar y sostener posturas al punto de forzar uno u otro o ambos con tal de hacerlos coincidir. Acordamos con Rivas Flores (2009:29) en la necesidad de llegar a una "violencia epistémica" que facilite el cuestionamiento de lo dicho y permita visibilizar los campos sociales, políticos, económicos y éticos en los que se han modelado. En las narrativas aparece también otra cuestión no menos importante, que es que la gran mayoría de las estudiantes que participaron reducen la experiencia de formación a lo que sucede en las prácticas realizadas en las instituciones del nivel, es decir que no aparece o se observa en muy pocos casos la incorporación en sus narrativas sobre el proceso de formación clases, lecturas, charlas dentro del grupo, o elaboración de trabajos prácticos, entre otras propuestas. En este trabajo manifestamos que las alumnas adjudican "a las instituciones de educación inicial, prácticamente la exclusividad en cuanto a la formación".

Haciendo entrevistas, luego de proponer a las estudiantes espacios de discusión con aportes de algunas lecturas y a fin de profundizar sobre lo que entienden por formación, algunas pasan de haber sostenido que se trata "de un proceso permanente de adquisición de herramientas, saberes, conocimientos, actitudes, etc.," a considerar que la formación se trata "de un encarnamiento porque hay hechos, personas, historias, emociones, etc. que nos atraviesan, nos marcan, es decir se hace experiencia" aunque todavía les resulta difícil hablar de "sus propias experiencias". En el período analizado para esta ponencia de alrededor de 70 producciones de narrativas solamente veinte han sido realizadas desde dentro, buceando hacia el interior de cada una, el resto recurre a conceptos teóricos, y se les dificulta hacerlo "desde su propia subjetividad, conectando y conectándose con y desde sus propias experiencias, emociones y sentimientos."

Del trabajo realizado hasta aquí, de las lecturas indispensables buscando pistas para comprender los complejos procesos de formación, de los diálogos con las estudiantes y las lecturas de sus narrativas claramente nos afirmamos en considerar a la formación docente como cuestión política, aspecto trabajado y presentado en el III Congreso Internacional de Educación realizado en la Pampa en el año 2015. Como sostiene Cullen (2007:35) "hay que trabajar en el cuidado de sí. El cuidado de sí es la resistencia al disciplinamiento de la singularidad que se configura, sobre todo, en la relación del saber con el poder." La actualidad demanda una "ciudadanía protagónica", basada en el ejercicio solidario del poder, la ética, y la sensibilidad frente a lo social, "ciudadanos cuestionadores, críticos y propositivos, capaces de demandar del Estado" Larrea (2008). Es en este sentido que la 
formación es política en tanto ofrece posibilidades de resistencia a los procesos de manipulación ideológica que se hace presente en diferentes medios e instituciones.

Las indagaciones realizadas durante todos estos años y la preocupación permanente por encontrar o crear algunas estrategias de intervención nos permitió advertir las complejidad de los procesos de formación en tanto deben impactar en cambios genuinos en las estudiantes para posibilitar otras formas de enseñanza que garanticen el respeto a los derechos de los niños y de las niñas y en las que la democratización de las relaciones pedagógicas les permita ejercer su quehacer atendiendo a las individualidades, a los potenciales exige elaborar algunos ejes sobre los que trabajar y que en el punto tres mencionaremos con más detalle. En primer lugar trabajar sobre los valores que estamos dispuestos a sostener y defender, valores identificados con aquéllos que permitan hacer de ésta, una sociedad más justa, en las que todos, sin ninguna distinción puedan ser realmente sujetos de derechos y en la que se asuman como sujetos políticos en la construcción de ese mundo. Valores que por otro lado, solamente se concretan en la vivencia, en la experiencia cotidiana, y que tienen que ver con la "conciencia histórica" de la que habla Alvarado (2009), refiere a ese posicionamiento que cada quien asume en el presente haciéndose cargo de su biografía, del camino recorrido y a la vez de sus futuros soñados, en cuanto "utopías realizables". También la desnaturalización de los procesos de socialización vividos por cada una y en ese proceso, la visibilización de las tramas desde o en las cuales nos vamos constituyendo como sujetos, sacar a la luz "nuestro colonialismo interno" (Cusicanqui, 2010). Red de relaciones, en muchos casos impregnadas de las lógicas de disciplinamiento propias de la escuela moderna en particular y de otras instituciones que se hicieron eco de las mismas como podría ser la familia.

Todo este recorrido no puede estar exento del análisis del contexto que refiere a la tierra que habitamos; situarnos en nuestro territorio y desde el mismo re pensarnos en diálogo intercultural, desde una especie de "domicilio existencial" al decir de Kusch (2007) ya que la cultura no es sólo el acervo espiritual que el grupo brinda a cada uno y que es aportado por la tradición, sino además el baluarte simbólico en el cual uno se refugia para defender la significación de su existencia. Cultura implica una defensa existencial frente a lo nuevo, porque si careciera uno de ella no tendría elementos para hacer frente a una novedad incomprensible (Kusch, 2007: 252).

Este anclaje en nuestro "suelo latinoamericano" necesariamente exige analizar las condiciones sociales, políticas y económicas que atraviesan los distintos niveles de la escolaridad y los procesos de formación docente y que hoy, más que nunca, están atravesados por el "discurso hegemónico de un modelo civilizatorio" (Lander, 2000) en referencia al neoliberalismo.

\section{3.- A modo de cierre (siempre provisorio)}

El Proyecto de Investigación, las preguntas que permanentemente nos hacemos, la sistematización de los datos para poder elaborar algunas respuestas, siempre abiertas y en constante revisión, las lecturas y re lecturas de quienes van pensando sobre similares preocupaciones nos ha permitido pensar algunas dimensiones para trabajar la formación docente a partir de lo que cada estudiante va "alumbrando" en sus procesos de escritura. Cuando hablamos de "dimensiones" lo hacemos pensando en algunos ejes o cuestiones centrales, en torno a las cuales pudiéramos organizar la información que se desprende de las reflexiones y narrativas de Ixs estudiantes, para desde allí proponer estrategias de 
acompañamiento que permitan ampliar y profundizar dichas reflexiones y que siempre van a girar en torno a las subjetividades, entendiendo por tal

un modo de hacer en el mundo. Una subjetividad es un modo de hacer con lo real. Llamaremos a esas prácticas sobre lo real operaciones y digamos con menor belleza que Buber que la subjetividad es la serie de operaciones realizadas para habitar un dispositivo, una situación, un mundo. La idea de que la subjetividad es una configuración práctica supone que la subjetividad es el conjunto de las operaciones realizadas, repetidas, inventadas. En tiempos institucionales los dispositivos obligan a los sujetos a ejecutar operaciones para permanecer en ellos. La subjetividad se instituye reproduciéndose, al mismo tiempo que se reproduce el dispositivo que instituye la subjetividad en cuestión; el dispositivo universitario reproduce subjetividad académica, el dispositivo escolar subjetividad pedagógica, el dispositivo familiar reproduce subjetividad paterno filial, etc." Corea y Lewkowicz (2005: 48)

Subjetividades, en este caso pedagógicas y en cuanto tal siguiendo a Cullen siempre "subjetividades ético políticas" y a las condiciones materiales y simbólicas en las que se van constituyendo. Lo que proponemos, insistimos en ello, son organizadores para las 'propuestas de intervención pedagógicas, no para trabajar sobre los estudiantes, sino sobre nuestro quehacer docente. Consideramos indispensable, para fortalecer el impacto de la formación en los graduados, proponer diversas estrategias que permitan abordar lo más exhaustivamente posible la complejidad del proceso en el que estamos comprometidos y asumiendo que lo inmediato para trabajar es el vínculo, el lazo que nos conecta y nos permite sentir que tenemos parte, que somos parte de un mundo en común, hemos elaborado las siguientes dimensiones que consideramos nos serán útiles para proponer en el próximo período académico dispositivos que enfoquen a las cuestiones que en cada caso mencionamos y según la peculiaridad de los procesos de los estudiantes.

Dimensión axiológica: la pensamos como aquélla que nuclea a los valores se van poniendo en juego en ese trabajo sobre uno mismo y como se han vivido a lo largo de cada historia y biografía escolar. Hemos trabajado mucho, sobre "el respeto" como valor, sin embargo hemos podido profundizar los sentidos que le otorgamos al mismo en función de las experiencias vividas que lo reducen a la obediencia, a la docilidad (que se traduce en diversas manifestaciones corporales) y que reflexionando desde las diversas lecturas nada tiene que ver con la misma palabra en contextos democráticos. En éstos el respeto tiene mucho que ver con la "dignidad humana" y por lo tanto con la libertad.

Así cómo el respeto aparece como un "valor" fuertemente incorporado, fundamentalmente por experiencias familiares y escolares pero con un sentido contrario a las actuales propuestas de socialización, entendemos que podríamos trabajar otros como "la libertad", "la vida", etc.

Dimensión epistemológica: no referimos a los saberes que la estudiante va construyendo en torno a sí mismo y a la relación de esos saberes con la formación docente y la profesión. Saberes que no es posible pensarlos por fuera de la relación saber /poder, y asumir que así como cada uno de nosotros fuimos configurados, dentro de estas relaciones de verdad, intervenimos igualmente en la conformación de otras subjetividades dando lugar a posibilidades de reproducción de similar lógica $o$ de transformación de las relaciones, posibilitando vínculos más horizontales, en el que sin perder el lugar de la autoridad, la misma no sea impedimento del desarrollo de los potenciales de cada quien y de la construcción de un mundo en el que todxs sin diferencias puedan ser parte y tomar parte. Esta 
dimensión surge de los análisis realizados por las estudiantes cuando pueden vincular sus prácticas pedagógicas en las instituciones de educación inicial con sus experiencias como alumnas en el sistema escolar y fundamentalmente en aquéllas situaciones en que el contraste entre lo que hacen en el aula y lo que dicen que hicieron es de tal magnitud que a las propias protagonistas sorprende.

Dimensión ontológica: como me descubro en ese "estar siendo" de los procesos de formación. No es posible pensar la formación docente descontextualizada. Nuestro piso firme es América Latina y en este sentido no podemos perder de vista que nuestro "ser" es siempre un "estar siendo", a partir de la interpelación que el "otro" nos hace. Y que en general hacemos a todo lo que "desde fuera" se nos impone. Dimensión ontológica que es precedida por la ética, en tanto el "rostro del otro" nos interpela exigiéndonos que no lo destruyamos.

Dimensión cultural: especialmente encuadrada en lo económico, lo social, lo político y ético que atraviesa todas las demás y tiene que ver con las tramas del contexto en la cual las otras dimensiones significados y sentidos en tanto se van constituyendo en la dinámica de la cultura que atraviesa a los sujetos en el día a día. Como lo planteamos antes se trata aquí de visibilizar las fuertes improntas que el capitalismo, el colonialismo y el patriarcado han depositado en todas las instituciones, en algunas con mayor presencia que en otras, y que intervienen en la constitución de las subjetividades.

Este año -2017- una estudiante a quien le ha costado mucho esfuerzo hacer este trabajo interior del que venimos hablando comienza su narrativa con la siguiente cita de Freire, la transcribimos porque damos fe de que ha hecho realidad las palabras del querido maestro:

"El hombre llega a ser sujeto por una reflexión sobre su situación, sobre su ambiente en concreto. Mientras más reflexiona sobre la realidad sobre su situación concreta, mas "emerge", plenamente consciente, comprometido, listo a intervenir respecto la realidad para cambiarla. (Paulo Freire). En la medida en que el hombre, integrándose en las condiciones de contexto de vida, reflexiona sobre ellas y aporta respuestas a los desafíos que le plantean, el hombre crea cultura."

Muchas gracias a todas las estudiantes del Taller de Integración, Investigación y Práctica III del profesorado en Educación Inicial que desde hace varios años vienen aportando a este proceso de formación que incansablemente llevamos adelante.

\section{Bibliografía}

Baquero, R; Diker, G. y Frigerio, G. (2007 (comps.). Las formas de lo escolar. Buenos Aires, Del Estante Editorial

Candau, J. (2001) Memoria e identidad. Buenos Aires: Del Sol.

Corea y Lewkowicz (2005) Pedagogía del aburrido. Escuelas destituidas, familias perplejas. Buenos Aires: Paidos pp $41-70$ Crujía

Cullen, C. (2009) Entrañas éticas de la identidad docente. Buenos Aires: La

(2007) Ciudadanía urbi et orbi en Carlos Cullen (compilador) $E$ malestar de la ciudadanía. Buenos Aires: La Crujía - Stella

Larrea, L. Pedagogía crítica para procesos de formación en ciudadanía y desarrollo local. En Paulo Freire. Contribuciones para la pedagogía. Moacir Gadotti, Margarita Victoria Gomez, Jason Mafra, Anderson Fernandes de Alencar (compiladores). CLACSO, Consejo Latinoamericano de Ciencias Sociales, Buenos Aires. Enero 2008. 


\section{http://bibliotecavirtual.clacso.org.ar/ar/libros/campus/freire/11Larrea.pdf}

Ferry, G. (1990). El trayecto de la formación. Los enseñantes entre la teoría y la práctica. México: Paidós Educador

Gerez Ambertin, M. (2009) Variantes de la soledad y la culpa en la clínica contemporánea, en 2do. Congreso Internacional de Investigación en Psicoanálisis, Derecho y Ciencias Sociales. Bs. As.: Letra Viva, 2009 pp 309 - 318 https://sites.google.com/a/fundpsicsigmundfreud.org/congreso-2009/

Lander, Edgardo. Ciencias sociales: saberes coloniales y eurocéntrico. En libro: La colonialidad del saber: eurocentrismo y ciencias sociales. Perspectivas Latinoamericanas. Edgardo Lander (comp.) CLACSO, Consejo Latinoamericano de Ciencias Sociales, Buenos Aires, Argentina. Julio de 2000. p. 246.

Disponible en la World Wide Web: http://bibliotecavirtual.clacso.org.ar/ar/libros/lander/lander1.rtf

Rivas Flores, J.I. (2012) Vida, Experiencia y educación: la biografía como estrategia de conocimiento, en I. Sverdlick, La investigación educativa. Una herramienta de conocimiento y de acción. Buenos Aires, Centro de Publicaciones Educativas y Material Didáctico

Rivas Flores, J.I. (2009). Narración, conocimiento y realidad. Un cambio de argumento en la investigación educativa. Rivas Flores y Herrera Pastor Voz y educación. La narrativa como enfoque de la realidad. Barcelona: Octaedro.

Rivas, J.I. y otros (2000). El Trabajo de los Docentes de Secundaria. Estudio Biográfico de su Cultura Profesional. Revista Interuniversitaria de Formación del Profesorado.

Santos, B. de Sousa. (2017). Democracia y transformación social. Bogotá: Siglo del Hombre Editores. Siglo XXI Editores

Santos, B. de Sousa (2009 Epistemologías del Sur. Perspectivas. México: Siglo XXI CLACSO

Zemelman Merino, H. (2010) Aspectos básicos de la propuesta de la conciencia histórica (o del presente potencial). México: IPECAL Instituto Pensamiento y cultura en América Latina.

Zemelman, H. (2005). Voluntad de conocer: El sujeto y su pensamiento en el paradigma crítico I. México: Antrhropos Editores. Centro de Investigaciones Humanísticas. Universidad Autónoma de Chiapas 